\title{
A Generalized Conjecture about Cutting a Polygon into Triangles of Equal Areas
}

\author{
S. Stein \\ Department of Mathematics, University of California at Davis, \\ 1 Shields Avenue, Davis, CA 95616-8633, USA \\ stein@math.ucdavis.edu
}

\begin{abstract}
In 1970 it was proved that a square cannot be cut into an odd number of triangles of equal areas. In 1990 it was proved that any centrally symmetric polygon has that property. In the present paper we propose a broad generalization, which would also imply that any polygon whose edges are parallel to the $x$ - or $y$-axes has that property. We prove that the generalization holds for polygons with at most six sides.
\end{abstract}

In 1970 it was proved that a square cannot be cut into an odd number of triangles of equal areas [1]. This result has been generalized to any centrally symmetric polygon [2], [3] and to any polyomino consisting of an odd number of squares [4]. We conjectured in [4] that the result holds for any polygon whose edges are parallel to the $x$ - or $y$-axes. We have also conjectured that the result holds for any polygon $P$ obtained from the square with vertices $(0,0),(1,0),(1,1),(0,1)$ by altering opposite edges in the same way so that $P$ still tiles the plane by translates by the vectors $(m, n)$, where $m$ and $n$ are integers.

The centrally symmetric polygons, the polygons with sides parallel to the axes, and the polygons obtained by distorting the square as just described are all cases of what we call special polygons, defined as follows. Let $P$ be a simply connected polygon in the $x y$-plane with an oriented boundary. Orient the edges on the boundary of $P$ to be consistent with the given orientation. Call two edges of $P$ equivalent if they are parallel. If the sum of the vectors in each equivalence class is the 0 -vector, we call $P$ a special polygon. We propose the following general conjecture: A special polygon cannot be cut into an odd number of triangles of equal areas.

There are no special polygons with three or five sides. The special polygons with four sides are parallelograms, hence affinely equivalent to a square; therefore the conjecture 


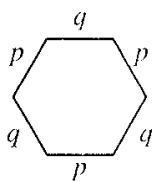

(a)

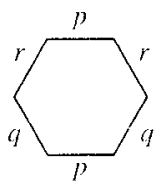

(b)

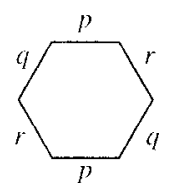

(c)

Fig. 1.

holds for them. There are three types of special polygons with six sides, schematically indicated in Fig. 1, where vectors labeled by the same letter are in fact parallel, as shown in Fig. 2. Type (c) is a centrally symmetric polygon. Type (a) is shaped like the letter L and type (b) is a dart, as shown in Fig. 2.
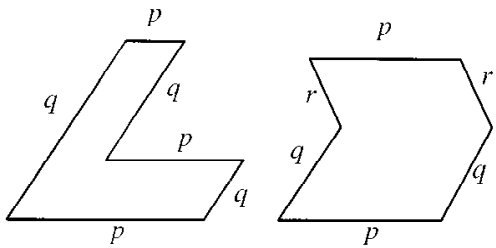

Fig. 2.

As mentioned, it is known that the conjecture already holds for type (c) polygons. We prove that it holds for the other two types as well.

The proofs use a 2-adic valuation on the reals. A function $\varphi: R \rightarrow R \cup\{\infty\}$ is a 2-adic valuation if $\varphi(x y)=\varphi(x)+\varphi(y), \varphi(x+y) \geq \min \{\varphi(x), \varphi(y)\}, \varphi(2)=1$, and $\varphi(0)=\infty$. It follows that $\varphi(1)=0$ and $\varphi(1 / x)=-\varphi(x)$. Moreover, for any odd integer $n, \varphi(n)=0$. In addition, if $\varphi(x)<\varphi(y)$ then $\varphi(x+y)=\varphi(x)$. For convenience we denote $\varphi(x)$ by $x^{\prime}$.

With the aid of a 2-adic valuation the points in the $x y$-plane can be divided into three types. If $x^{\prime}>0$ and $y^{\prime}>0,(x, y)$ is of type 0 and is denoted $P_{0}$. If $x^{\prime} \leq 0$ and $x^{\prime} \leq y^{\prime}$, it is of type 1 and is denoted $P_{1}$. If $y^{\prime} \leq 0$ and $y^{\prime}<x^{\prime}$, it is of type 2 and is denoted $P_{2}$.

A line segment whose ends are a $P_{0}$ and a $P_{1}$ is called complete. A polygon whose border contains an odd number of complete edges is also called complete. If a complete polygon of area $A$ is cut into $m$ triangles of equal areas, then $m^{\prime} \geq(2 A)^{\prime}=1+A^{\prime}$. In particular, if $A^{\prime}>-1$, then $m$ is even [1]-[4].

A tiling of a polygon by triangles of equal areas we call an equidissection. An equidissection by an odd number of triangles is called an odd equidissection.

Theorem 1. A special polygon of type (a) has no odd equidissection.

Proof. Assume that such a polygon, situated in the $x y$-plane, is tiled by $m$ triangles of equal areas. Label its vertices in order around the boundary $Q_{1}, Q_{2}, Q_{3}, Q_{4}, Q_{5}, Q_{6}$. Each triplet of three consecutive vertices, $Q_{i}, Q_{i+1}, Q_{i+2}$, where the indices are viewed modulo 6, determines a triangle of area $A_{i}$. Let $Q_{j}, Q_{j+1}, Q_{j+2}$ be a triplet for which $A_{j}^{\prime}$ is a minimum. 
Let $T$ be the affine transformation such that $T\left(Q_{j}\right)=(1,0), T\left(Q_{j+1}\right)=(0,0)$, and $T\left(Q_{j+2}\right)=(0,1)$. Let $R$ be the image of the given polygon under $T$. It is L-shaped, with all its edges parallel to the axes. Let the vertex opposite $(0,0)$ be $(a, b)$. The remaining two vertices then are $(1, b)$ and $(a, 1)$. Figure 3 displays the labeling of $R$.

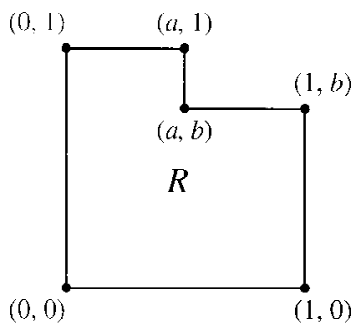

Fig. 3.

The area of the triangle with vertices $(0,1),(0,0)$, and $(1,0)$ is $1 / 2$. The area of the triangle with vertices $(0,0),(1,0)$, and $(1, b)$ is $b / 2$. The area of the triangle with vertices $(0,0),(0,1)$, and $(a, 1)$ is $a / 2$. Since $T$ preserves ratios of areas, it follows that $(b / 2)^{\prime}$ and $(a / 2)^{\prime}$ are at least $(1 / 2)^{\prime}$. Thus $a^{\prime}$ and $b^{\prime}$ are nonnegative.

It follows that (area of $R)^{\prime}$, which is $(a+b-a b)^{\prime}$, is also nonnegative. The types of four of the six vertices are determined, as shown in Fig. 4. Only the types of the vertices $P=(a, 1)$ and $Q=(a, b)$ are not.

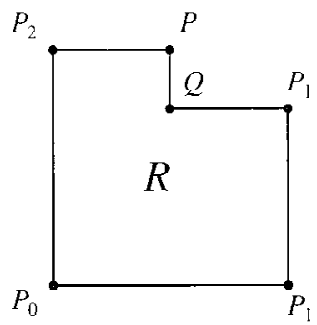

Fig. 4.

The only case when the labeling is not complete occurs when $P$ is a $P_{2}$ and $Q$ is a $P_{0}$. Consider this case, which corresponds to $a^{\prime}>0$ and $b^{\prime}>0$.

Assume that $b^{\prime} \leq a^{\prime}$. Introduce the linear mapping $(x, y) \rightarrow(x, y / b)$ and let $S$ be the image of $R$ under this mapping. Figure 5 shows $S$, which is complete.

Since the determinant of the mapping is $1 / b$ the area of $S$ is

$$
\frac{a+b-a b}{b}=\frac{a}{b}+1-a
$$

Hence (area of $S)^{\prime} \geq 0$. Thus $m$ is even and the theorem is proved in this case. 


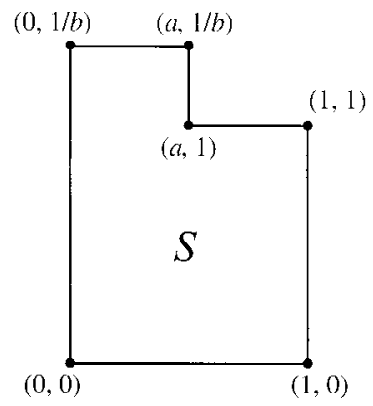

Fig. 5.

The case when $a^{\prime}<b^{\prime}$ follows immediately by symmetry (or by using the linear mapping $(x, y) \rightarrow(x / a, y))$. This completes the proof.

Note that Theorem 1 implies that any polygon with at most six sides whose sides are parallel to the axes has no odd equidissection.

Theorem 2. A special polygon of type (b) has no odd equidissection.

Proof. Any such polygon is affinely equivalent to a polygon $R$ with the vertices $(0,0)$, $(1,0),(1,1),(0,1),(a, b),(1+a, b)$, shown in Fig. $6 . R$ has area 1 . Let $P=(a, b)$. If $P$ is a $P_{0}$ or a $P_{2}$, the labeling is complete and the theorem follows. So consider the case when $P$ is a $P_{1}$, that is, when $a^{\prime} \leq 0$ and $a^{\prime} \leq b^{\prime}$.

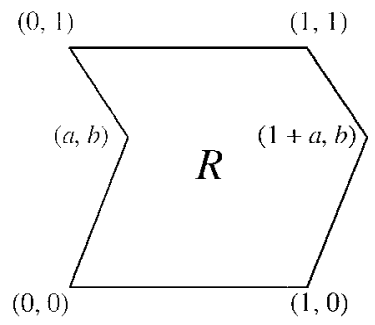

Fig. 6.

If $a^{\prime}=b^{\prime}$, consider the image of $R$ under the reflection $(x, y) \rightarrow(y, x)$. The image, $S$, shown in Fig. 7, is complete. Since the area of $S$ is 1 , the theorem holds in this case.

Next consider the case $a^{\prime}<b^{\prime}$. Apply the linear mapping that takes $(1,0)$ to $(1,0)$ and $(a, b)$ to $(0,1)$, namely

$$
(x, y) \rightarrow\left(x-\frac{a}{b} y, \frac{1}{b} y\right) .
$$

This mapping has determinant $1 / b$. The image of $R$ is the polygon $T$ shown in Fig. 8 . 


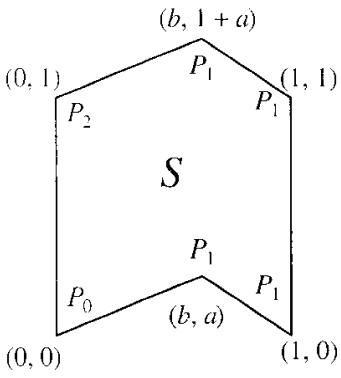

Fig. 7.

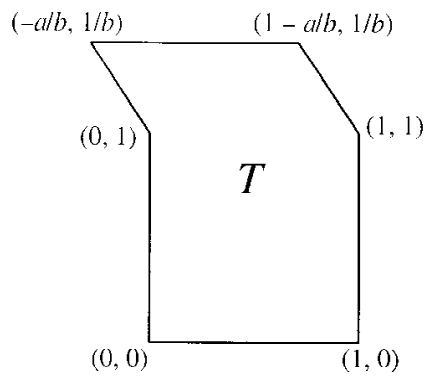

Fig. 8.

Since $(a / b)^{\prime}=a^{\prime}-b^{\prime}<0, T$ is complete. Letting the number of triangles in a dissection of $T$ be $m$, we therefore have

$$
m^{\prime} \geq 2(\operatorname{area} \text { of } T)^{\prime}=\left(2\left(\frac{1}{b}\right)\right)^{\prime}=1-b^{\prime} .
$$

If $b^{\prime}<1$ it follows that $m$ is even.

Consider finally the case when $a^{\prime} \leq 0$ and $b^{\prime} \geq 1$. Reflect $R$ in the line $y=\frac{1}{2}$ by means of the affine mapping $(x, y) \rightarrow(x, 1-y)$. The image of $(a, b)$ is $(a, 1-b)$. Since $a^{\prime} \leq 0$ and $(1-b)^{\prime}=0$, we have a case included in the case $a^{\prime} \leq 0, b^{\prime}<1$. This concludes the proof.

Note that Theorem 2 implies that any hexagon obtained by distorting a square in the manner described earlier has no odd equidissection.

Similar arguments show that the general conjecture holds for several families of special polygons with more than six sides.

\section{References}

1. P. Monsky, On dividing a square into triangles, Amer. Math. Monthly 77 (1970), 163-164.

2. P. Monsky, A conjecture of Stein on plane dissections, Math. Z. 205 (1990), 583-592.

3. S. Stein, Equidissections of centrally symmetric octagons, Aequationes Math. 37 (1989), 113-118.

4. S. Stein, Cutting a polyomino into triangles of equal areas, Amer. Math. Monthly 106 (1999), 255-257.

Received November 24, 1999, and in revised form January 28, 2000. Online publication May 3, 2000. 